\title{
Design and Development of an Educational Desktop Robot $\mathbf{R}^{3} \mathbf{D}$
}

\author{
OSMAN NURI SAHIN, ${ }^{1,2}$ EMRE UZUNOGLU, ${ }^{1,2}$ ENVER TATLICIOGLU, ${ }^{3}$ M.I. CAN DEDE ${ }^{1}$ \\ ${ }^{1}$ Department of Mechanical Engineering, Izmir Institute of Technology, Izmir 35430, Turkey \\ ${ }^{2}$ NLFD Technology. Inc., Izmir Technology Development Zone. Inc., A6 No: 5/H, Izmir Institute of Technology Technopark, \\ Izmir 35430, Turkey \\ ${ }^{3}$ Department of Electrical and Electronics Engineering, Izmir Institute of Technology, Izmir 35430, Turkey
}

Received 16 November 2016; accepted 21 December 2016

\begin{abstract}
Robotic desktop devices have been used for academic purposes for a variety of investigation and development studies. Desktop devices for academic/educational purposes have been highly anticipated especially in the fields of haptics, teleoperation systems, and control studies. This paper's motivation is to present the steps of designing, manufacturing, and implementing of Educational Desktop Robot $\mathrm{R}^{3} \mathrm{D}$ to be used for haptics, teleoperation, and redundancy control studies. The design, manufacturing details, kinematic, and dynamic model of the robot are described in the manuscript. Additionally, a case study is carried out for end effector control in task space is given and the results are shared. (c) 2017 Wiley Periodicals, Inc. Comput Appl Eng Educ 25:222-229, 2017; View this article online at wileyonlinelibrary.com/journal/cae; DOI 10.1002/cae.21792
\end{abstract}

Keywords: design of a robot manipulator; control; task space

\section{INTRODUCTION}

In engineering education, laboratory experimental setups are useful as educational tools just as they are essential evaluation platforms for testing theoretical methods. It is acknowledged in the literature that developing a laboratory experimental setup usually costs much less compared to commercially available setups, and researchers and students gain significant experience in design and construction of real systems [1-18]. Among those laboratory experimental setups robot manipulators are considered as good examples of multiple-input multiple-output nonlinear systems that can be utilized for both control and robotics education. In addition to their inter/multi-disciplinary usage, robot manipulators can be utilized in both undergraduate and graduate level courses. Introduction to robotics and mechatronics courses and such like can be given as an example for undergraduate level courses. More than this, graduate level course such as non-linear control, redundant control, haptics, and teleoperation and graduate level

Correspondence to E. Tatlicioglu (envertatlicioglu@iyte.edu.tr).

(C) 2017 Wiley Periodicals, Inc. research in this areas are possible application area of the proposed system. According to the authors' best knowledge, there are only a few past works fully dedicated to design and development of a robot manipulator [12-18]. Some line of past research has focused on designing mobile robots [12]. In Ref. [13], an articulated desktop robotic device called Rhino XR4 is proposed to be used as an educational robotic workstation with graphical user interface for students to perform experiments. Moreover, in the literature, a variety of robot design and development studies are presented for haptic applications [14-18]. The results in Refs. [13-18] emphasize the importance of programming as an important part of robotics.

In this study, an $\mathrm{R}^{3} \mathrm{D}$ robot manipulator which is a desktop device inspired from the lack of low-cost, easy-to-use robotic systems is developed. The robot manipulator works in the planar workspace and has three Degrees of Freedom (DoF) articulated structure. The main motivation behind this study is to gain the experience in designing a robot manipulator. Another important motivation of developing this robot is to apply linear and nonlinear control strategies. Firstly, by utilizing the physical properties of the robot manipulator a kinematic model can be obtained, which is followed by deriving a dynamic model via using energy based methods [19-21]. When the control problem is to drive the joint 
positions to desired constant or time-varying values (i.e., joint space control) both linear (i.e., proportional derivative integral) controllers and nonlinear controllers can be applied [19]. On the other hand, when the control objective is to move the end-effector of the robot manipulator to a desired pose (i.e., task space control) two common methods are preferred in the literature. One method converts the desired pose into desired joint positions via utilizing inverse kinematics at the position level and then makes use of joint space control $[20,21]$. The other method makes use of inverse kinematics at the velocity level and nonlinear task space controllers can be designed [20,21]. In task space control applications, when the desired pose requires only positioning of the end-effector (i.e., the orientation of the end-effector is not driven to a desired value), the robot manipulator becomes kinematically redundant [22]. Similar to most robot manipulator designs, $R^{3} D$ is not equipped with joint velocity measurement tools. On the other hand, several control algorithms require joint velocities. To overcome this restriction, linear or nonlinear velocity observers can be applied [19,23]. Apart from the control applications, $\mathrm{R}^{3} \mathrm{D}$ can be configured as a master haptic device (force-reflecting device) which can be adapted for teleoperation systems or virtual reality scenarios.

In this paper, design and manufacturing phases of the educational desktop robot $\mathrm{R}^{3} \mathrm{D}$ are presented. Firstly, the design of the mechanism that includes mechanical structure, electromechanical hardware, and controller interface are described. The mechanical structures are manufactured and assembled with electromechanical parts by the members of Robotics and Control Laboratories of Izmir Institute of Technology. Due to ease of use, Matlab Simulink environment is chosen for robot control and also as the user interface. Forward and inverse kinematic models and dynamic model of the robot manipulator are then obtained. Finally, a task-space controller is designed [24] and experiment results are presented to demonstrate the performance of the overall system.

\section{DESIGN OF $\mathbf{R}^{3} D$}

The overall mechanical system consists of structural base, link frames, actuators, and sensors. The planar robot has articulated structure which consists of three DoF, or equivalently, three actuators that works in the same plane. The manufactured and assembled device is shown in Figure 1, where the base frame links and end-effector are labeled. Base frame is the structural component where the first link is connected to and constructed inside an aluminum casing. Consecutive links and joints have identical design structure as can be seen from Figure 1. Some basic specifications of $R^{3} \mathrm{D}$ are summarized in Table 1 .

The mechanism has three joints which are actuated using direct drive DC motors. The base frame, illustrated in Figure 2, represents the fixed point of the mechanism and also designed to counter the weights of the links. The first actuator which is the only one that is stationary is assembled in the aluminum box (base) that has dimensions of $132.5 \times 132.5 \times 206 \mathrm{~mm}$. The sensors are connected to the rear shaft of the motors using $3 \mathrm{D}$ printed parts assembled to the rear shaft of the motors. For rigidity and better performance, proper bedding and power transmission components are required. The power transmission with the main shaft is done by spring coupling presented in Figure 2. Bearing house is used to mount the bearings and to create the necessary bedding for first shaft. Two bearings are integrated to withstand axial and radial loads.

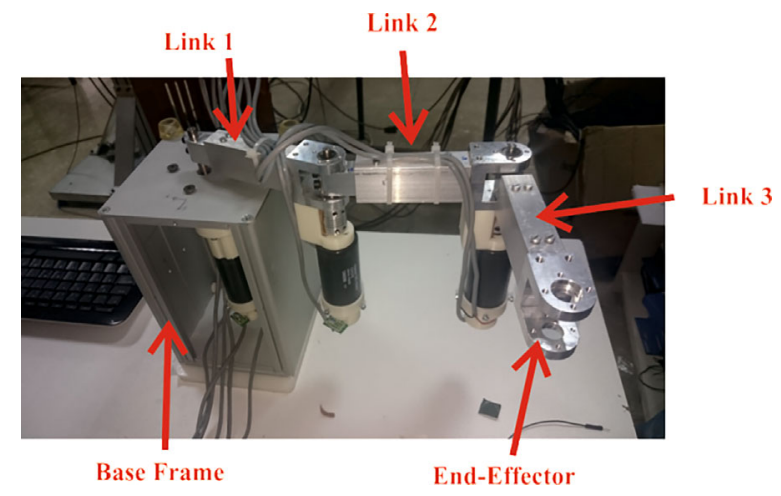

Figure 1 Educational desktop robot $\mathrm{R}^{3} \mathrm{D}$.

Table 1 Basic Specifications of $R^{3} D$

\begin{tabular}{lc}
\hline Characteristic & Value \\
\hline Efficient workspace & $210 \times 297$ millimeters \\
Link lengths & $127 \mathrm{~mm}$ \\
Joint limits & 210 degrees \\
Power supply & 24 VDC $10 \mathrm{~A}$ \\
Accuracy & $< \pm 1 \mathrm{~mm}$ \\
\hline
\end{tabular}

Links and parts of the joints are manufactured with CNC machining from aluminum. The motor and encoder connection parts are printed in $3 \mathrm{D}$ printer using $\mathrm{ABS}+$ plastic. The magnet of the magnetic encoder is shrink-fitted to the rear shaft of the motor via 3D printed part. In Figure 3, joint part 1 and joint part 2 represent the two parts that connects two consecutive links. Power transmission is realized similar to the design in the base frame in which a coupling and two bearings were used as shown in Figure 3.

The electromechanical parts of the robot manipulator are DC motors and their drivers, and magnetic encoders (see Figs. 2 and 3). Each DoF is actuated by using direct drive motors. Direct drive motor technology is preferred for the actuators

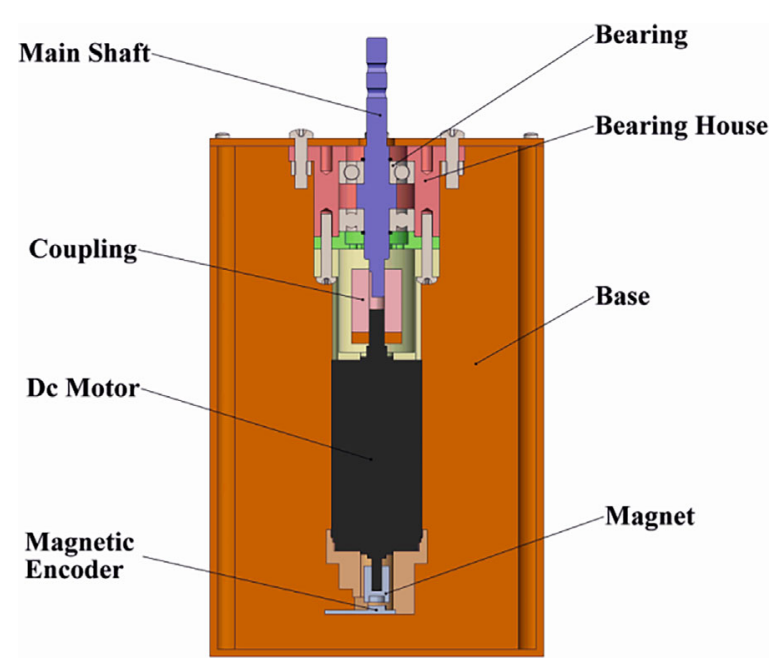

Figure 2 Base frame design. 


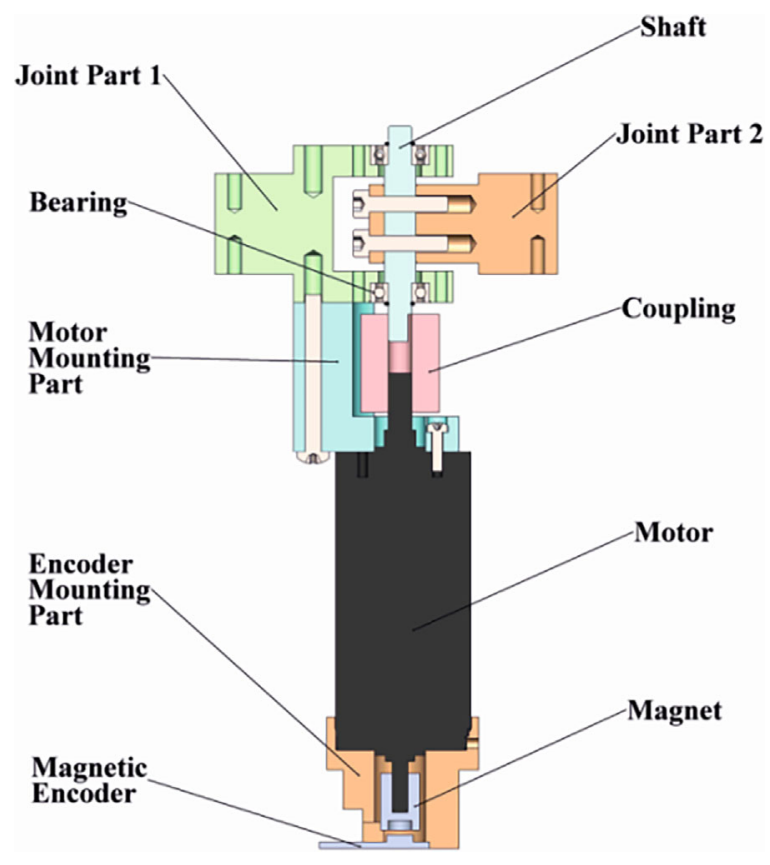

Figure 3 Link frame design.

simply because the robot manipulator is desired to be used in haptic and teleoperation experiments. E137576 Maxon motors are chosen as actuators where their characteristics are given in Table 2. Maxon Escon 36/2 DC, 4-Q Servocontroller enables to drive the motors which can support a maximum power of 72 Watts. These drivers are programmable and support to be operated in both current and speed mode, which provides flexibility to implement different control strategies. The Arms AS5045 magnetic rotary encoders were used as position sensors which deliver outputs as A, B, and I phases of incremental encoder with a 4096 bit resolution. compiled, this model is connected through this kernel to the DAQ card for real-time information exchange. In this setting, the joint actuator commands are transmitted to the motor drivers with analog signals as torque demands and encoder signals are received as quadrature counter inputs. The data transmission from PC, signal inputs, and outputs, are carried out with Humusoft MF 624DAQ (data acquisition) PCI card. The signal flow scheme between the peripherals is illustrated in Figure 4, which comprises a PC, PCI DAQ, actuator, and drivers.

\section{MODELING OF $R^{3} D$}

In this section, forward and inverse kinematic models of $R^{3} D$ are first obtained and next its dynamic model is derived. The basic kinematic sketch of the robot manipulator is illustrated in Figure 5 where modelling parameters are given in Table 3 .

Forward kinematics is commonly used to calculate position and orientation of the end-effector by using angular joint positions [19-21]. Making use of the link parameters denoted in Figure 5, forward kinematics equations for task space position are formulated as shown below

$$
\left[\begin{array}{l}
x \\
y \\
\gamma
\end{array}\right]=\left[\begin{array}{c}
a_{1} \cos \left(\theta_{1}\right)+a_{2} \cos \left(\theta_{1}+\theta_{2}\right)+a_{3} \cos \left(\theta_{1}+\theta_{2}+\theta_{3}\right) \\
a_{1} \sin \left(\theta_{1}\right)+a_{2} \sin \left(\theta_{1}+\theta_{2}\right)+a_{3} \sin \left(\theta_{1}+\theta_{2}+\theta_{3}\right) \\
\theta_{1}+\theta_{2}+\theta_{3}
\end{array}\right]
$$

where $x(t)$ and $y(t)$ denote the position, and $\gamma(t)$ is the orientation of the end-effector with respect to the base frame.

Differentiating (1) with Respect to Time Results in the Velocity Kinematics

$$
\left[\begin{array}{c}
\dot{x} \\
\dot{y} \\
\dot{\gamma}
\end{array}\right]=\widehat{j}\left[\begin{array}{l}
\dot{\theta}_{1} \\
\dot{\theta}_{2} \\
\dot{\theta}_{3}
\end{array}\right]
$$

where $\widehat{J}(\theta) \in \mathbb{R}^{3 \times 3}$ is Jacobian matrix that can be obtained as

$$
\widehat{j}=\left[\begin{array}{ccc}
-a_{1} \sin \left(\theta_{1}\right)-a_{2} \sin \left(\theta_{1}+\theta_{2}\right)-a_{3} \sin \left(\theta_{1}+\theta_{2}+\theta_{3}\right) & -a_{2} \sin \left(\theta_{1}+\theta_{2}\right)-a_{3} \sin \left(\theta_{1}+\theta_{2}+\theta_{3}\right) & -a_{3} \sin \left(\theta_{1}+\theta_{2}+\theta_{3}\right) \\
a_{1} \cos \left(\theta_{1}\right)+a_{2} \cos \left(\theta_{1}+\theta_{2}\right)+a_{3} \cos \left(\theta_{1}+\theta_{2}+\theta_{3}\right) & a_{2} \cos \left(\theta_{1}+\theta_{2}\right)+a_{3} \cos \left(\theta_{1}+\theta_{2}+\theta_{3}\right) & a_{3} \cos \left(\theta_{1}+\theta_{2}+\theta_{3}\right) \\
1 & 1 & 1
\end{array}\right]
$$

The control algorithms are developed in a PC using Matlab Simulink software package. The interface to receive sensor data from a data acquisition (DAQ) card and send torque demands to the motor drivers via a DAQ card is the Simulink Desktop Real-Time blocks, which can be configured for a variety of commercially available DAQ cards. Matlab provides a real-time kernel for executing Simulink models in real time and when this Simulink Model with the control algorithm and Simulink Desktop Real-Time interface blocks is

Table 2 Characteristics of E37576 Maxon Motor

\begin{tabular}{lc}
\hline Characteristic & Value \\
\hline Nominal voltage & $24 \mathrm{VDC}$ \\
Torque constant & $36.4 \mathrm{mNm} / \mathrm{A}$ \\
Speed constant & $263 \mathrm{rpm} / \mathrm{V}$ \\
Nominal speed & $5530 \mathrm{rpm}$ \\
Nominal torque & $78.2 \mathrm{mNm}$ \\
\hline
\end{tabular}

Given the end-effector position and orientation, angular joint position can be obtained via utilizing inverse kinematics calculations [19-21]. Specifically, given $x(t), y(t)$, and $\gamma(t)$, two known auxiliary variables, denoted by $x^{*}(t)$ and $y^{*}(t)$, can be calculated as

$$
\begin{gathered}
x^{*}=x-a_{3} \cos (\gamma)=a_{1} \cos \left(\theta_{1}\right)+a_{2} \cos \left(\theta_{1}+\theta_{2}\right) \\
y^{*}=y-a_{3} \sin (\gamma)=a_{1} \sin \left(\theta_{1}\right)+a_{2} \sin \left(\theta_{1}+\theta_{2}\right)
\end{gathered}
$$

The expressions in (4) are first squared and summed and then making use of trigonometric relations following expressions can be obtained

$$
\cos \left(\theta_{2}\right)=\frac{\left(x^{*}\right)^{2}+\left(y^{*}\right)^{2}-a_{1}^{2}-a_{2}^{2}}{2 a_{1} a_{2}}, \sin \left(\theta_{2}\right)= \pm \sqrt{1-\cos ^{2}\left(\theta_{2}\right)}
$$

from which two solutions of $\theta_{2}$ can be obtained as

$$
\theta_{2}=\operatorname{atan}_{2}\left( \pm \sin \left(\theta_{2}\right), \cos \left(\theta_{2}\right)\right)
$$




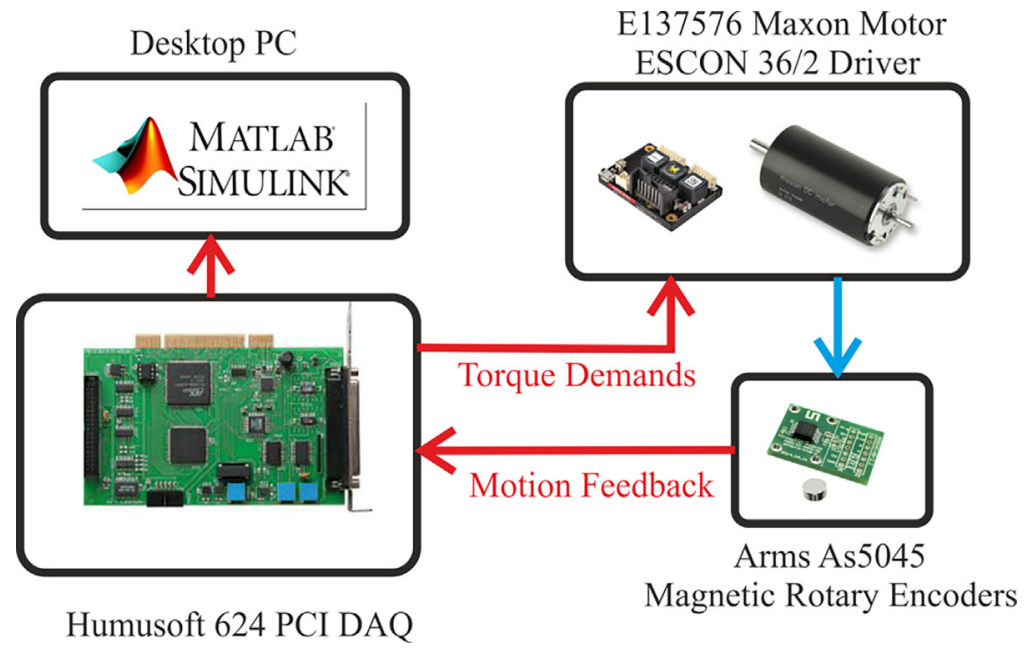

Figure 4 Signal flow scheme between the peripherals.

The angular position of the first joint can be calculated as

$$
\theta_{1}=\operatorname{atan}_{2}\left(\sin \left(\theta_{1}\right), \cos \left(\theta_{1}\right)\right)
$$

where

$$
\begin{aligned}
& \sin \left(\theta_{1}\right)=\frac{y^{*}\left(a_{1}+a_{2} \cos \left(\theta_{2}\right)\right)-x^{*} a_{2} \sin \left(\theta_{2}\right)}{\mathrm{a}_{1}^{2}+\mathrm{a}_{2}^{2}+2 \mathrm{a}_{1} \mathrm{a}_{2} \cos \left(\theta_{2}\right)}, \\
& \cos \left(\theta_{1}\right)=\frac{x^{*}\left(a_{1}+a_{2} \cos \left(\theta_{2}\right)\right)+y^{*} a_{2} \sin \left(\theta_{2}\right)}{\mathrm{a}_{1}^{2}+\mathrm{a}_{2}^{2}+2 \mathrm{a}_{1} \mathrm{a}_{2} \cos \left(\theta_{2}\right)}
\end{aligned}
$$

Finally, the angular position of the third joint is calculated as

$$
\theta_{3}=\gamma-\theta_{2}-\theta_{1}
$$

When the desired end-effector pose requires only positioning of the end-effector (i.e., the orientation of the end-effector is not driven to a desired value), the Jacobian matrix, denoted by (8) $\widehat{J}_{r}(\theta) \in \mathbb{R}^{2 \times 3}$, is obtained as

$$
\widehat{j}_{\mathrm{r}}=\left[\begin{array}{ccc}
-a_{1} \sin \left(\theta_{1}\right)-a_{2} \sin \left(\theta_{1}+\theta_{2}\right)-a_{3} \sin \left(\theta_{1}+\theta_{2}+\theta_{3}\right) & -a_{2} \sin \left(\theta_{1}+\theta_{2}\right)-a_{3} \sin \left(\theta_{1}+\theta_{2}+\theta_{3}\right) & -a_{3} \sin \left(\theta_{1}+\theta_{2}+\theta_{3}\right) \\
a_{1} \cos \left(\theta_{1}\right)+a_{2} \cos \left(\theta_{1}+\theta_{2}\right)+a_{3} \cos \left(\theta_{1}+\theta_{2}+\theta_{3}\right) & a_{2} \cos \left(\theta_{1}+\theta_{2}\right)+a_{3} \cos \left(\theta_{1}+\theta_{2}+\theta_{3}\right) & a_{3} \cos \left(\theta_{1}+\theta_{2}+\theta_{3}\right) \\
1 & 1 & 1
\end{array}\right]
$$

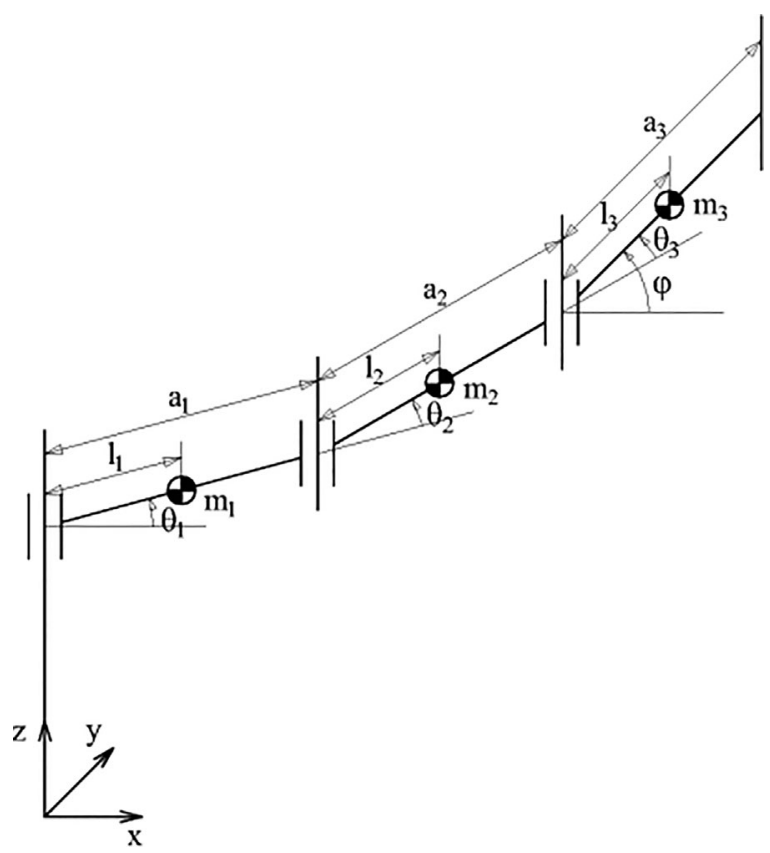

Figure 5 Kinematic sketch of $R^{3} D$.
The joint space dynamic model of the robot manipulator is developed using the Euler-Lagrange equations of motion and can be represented in matrix form as

$$
\left[\begin{array}{l}
\tau_{1} \\
\tau_{2} \\
\tau_{3}
\end{array}\right]=\mathrm{M}\left[\begin{array}{l}
\ddot{\theta}_{1} \\
\ddot{\theta}_{2} \\
\ddot{\theta}_{3}
\end{array}\right]+\mathrm{N}\left[\begin{array}{c}
\dot{\theta}_{1} \\
\dot{\theta}_{2} \\
\dot{\theta}_{3}
\end{array}\right]
$$

where $M(\theta) \in \mathbb{R}^{3 \times 3}$ is the positive definite, symmetric inertia matrix and $N(\theta, \dot{\theta}) \in \mathbb{R}^{3 \times 3}$ denotes Coriolis and centripetal effects and are of the following form

Table 3 Modelling Parameters

\begin{tabular}{lc}
\hline Parameter & Value \\
\hline Link lengths $\left(\mathrm{a}_{1}, \mathrm{a}_{2}\right.$, and $\left.\mathrm{a}_{3}\right)$ & $127 \mathrm{~mm}$ \\
Mass center $\left(\mathrm{l}_{1}, \mathrm{l}_{2}\right)$ & $106.20 \mathrm{~mm}$ \\
Mass center $\left(\mathrm{l}_{3}\right)$ & $71.58 \mathrm{~mm}$ \\
Mass $\mathrm{m}_{1}, \mathrm{~m}_{2}$ & $595.96 \mathrm{~g}$ \\
Mass $\mathrm{m}_{3}$ & $160.48 \mathrm{~g}$ \\
Inertia $\mathrm{I}_{1}$ and $\mathrm{I}_{2}$ & $6.729207 \mathrm{gm}^{2}$ \\
Inertia $\mathrm{I}_{3}$ & $0.82960524 \mathrm{gm}^{2}$ \\
\hline
\end{tabular}




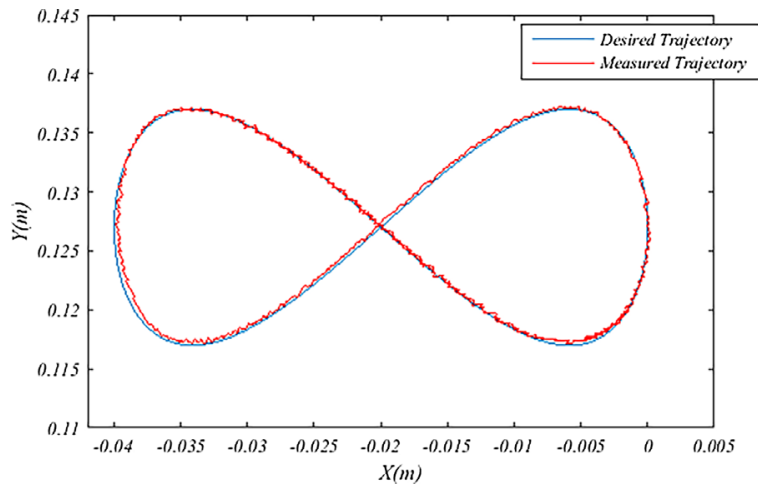

Figure 6 End-effector trajectory tracking performance.

$$
\begin{aligned}
\mathbf{M} & =\left[\begin{array}{lll}
\mathrm{m}_{11} & \mathrm{~m}_{12} & \mathrm{~m}_{13} \\
\mathrm{~m}_{12} & \mathrm{~m}_{22} & \mathrm{~m}_{23} \\
\mathrm{~m}_{13} & \mathrm{~m}_{23} & \mathrm{~m}_{33}
\end{array}\right], \\
\mathbf{N} & =\left[\begin{array}{ccc}
\gamma_{112} \dot{\theta}_{2}+\gamma_{113} \dot{\theta}_{3} & B_{12} \dot{\theta}_{2}+\gamma_{123} \dot{\theta}_{3} & B_{13} \dot{\theta}_{3} \\
B_{21} \dot{\theta}_{2} & \gamma_{223} \dot{\theta}_{3} & B_{23} \dot{\theta}_{3}+\gamma_{213} \dot{\theta}_{1} \\
B_{31} \dot{\theta}_{1} & B_{32} \dot{\theta}_{2}+\gamma_{312} \dot{\theta}_{1} & 0
\end{array}\right]
\end{aligned}
$$

It should be noted that there are no counter-mass and gravitational effects acting on robot. The entries of the dynamic modelling matrices are given the Appendix.

\section{EXPERIMENT RESULTS}

In this section, performance of the $R^{3} D$ is evaluated with a task space controller in Refs. [25,26]. The exact model knowledge controller is implemented where kinematic and dynamic models presented in previous sections are utilized. The tracking control objective is quantified via the task-space tracking error, denoted by $e(t) \in \mathbb{R}^{2}$, defined as

$$
e=X_{d}-X
$$

where $X(t)=\left[\begin{array}{ll}x(t) & y(t)\end{array}\right]^{T} \in \mathbb{R}^{2}$ is the end-effector position and $X_{d}(t) \in \mathbb{R}^{2}$ is the desired end-effector position. The end-effector position $X(t)$ is calculated by using the joint positions obtained from encoder measurements along with the forward kinematics.

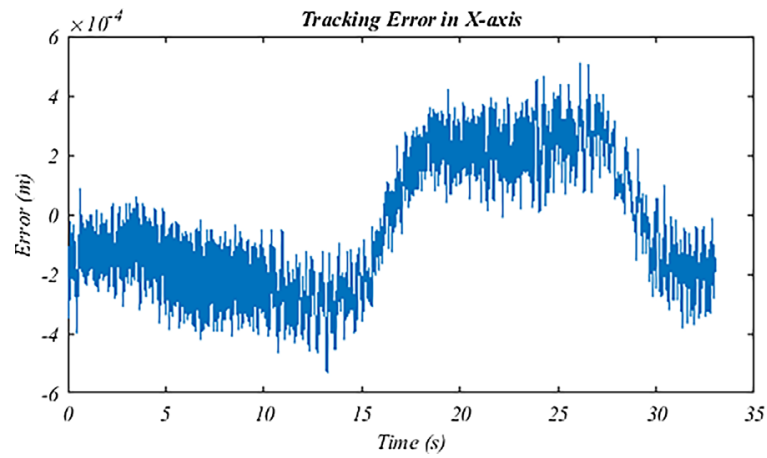

Figure $7 \quad \mathrm{X}$-axis error.

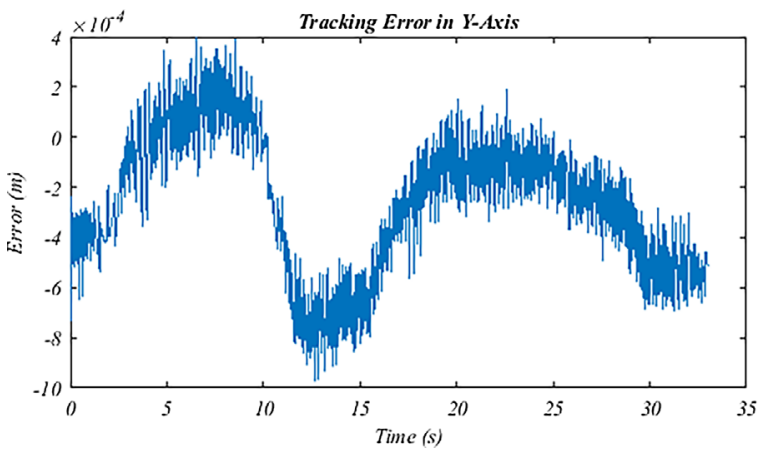

Figure 8 Y-axis error.

Since there is no desired end-effector orientation given, for this task, the robot is kinematically redundant.

The control input torque is designed as [27]

$$
\begin{aligned}
\tau= & J^{T} e+K_{r}\left(\dot{X}_{d}+K_{e} e-\dot{\theta}\right)+\mathrm{M} \\
& {\left[\dot{J}^{+}\left(\dot{X}_{d}+K_{e} e\right)+J^{+}\left(\ddot{X}_{d}+K_{e} \dot{e}\right)\right]+N J^{+}\left(\dot{X}_{d}+K_{e} \mathrm{e}\right) }
\end{aligned}
$$

where $K_{e} \in \mathbb{R}^{2 \times 2}$ and $K_{r} \in \mathbb{R}^{3 \times 3}$ are positive-definite diagonal control gain matrices, and $J^{+}(\theta) \in \mathbb{R}^{3 \times 2}$ is the pseudo-inverse of $\widehat{J}_{r}(\theta)$ defined as

$$
J^{+}=\widehat{J}_{r}^{T}\left(\widehat{J}_{r} \widehat{J}_{r}^{T}\right)^{-1}
$$

An infinity-like desired task space trajectory was designed as given in the Equations below with desired position, $X_{d}$, velocity, $\dot{X}_{d}$, and acceleration, $\ddot{X}_{d}$ as a function of time, $t$. The maximum task space velocity achieved during the given motion is $8 \mathrm{~mm} / \mathrm{s}$ with a maximum acceleration of $3.2 \mathrm{~mm} / \mathrm{s}^{2}$.

$$
\begin{gathered}
X_{d}=\left[\begin{array}{c}
-0.02+0.02 \cos (0.4 t) \\
0.127-0.01 \cos (0.4 t)
\end{array}\right] \\
\dot{X}_{d}=\left[\begin{array}{c}
-0.08 \sin (0.4 t) \\
-0.04 \cos (0.4 t)
\end{array}\right] \\
X_{d}^{*}=\left[\begin{array}{c}
-0.0032 \cos (0.4 t) \\
0.0016 \sin (0.4 t)
\end{array}\right]
\end{gathered}
$$

The initial joint positions were $\theta_{1}=0^{\circ}, \theta_{2}=90^{\circ}$, $\theta_{3}=90^{\circ}$ and the control gain matrices are chosen via trial-anderror as $K_{r}=\operatorname{diag}\{55,55,55\}$ and $K_{e}=\operatorname{diag}\{65,65\}$.

The results are presented in Figures 6-8. In Figure 6, desired task-space trajectory and end-effector position are demonstrated. The tracking errors in $x$ - and $y$-axis are shown in Figures 7 and 8, respectively. In each axes, error between $\pm 1 \mathrm{~mm}$ is observed at steady-state.

\section{CONCLUSIONS}

An educational desktop robot, $\mathrm{R}^{3} \mathrm{D}$, is developed for experimenting control designs. The mechanical structure is designed to work in plane, $2 \mathrm{D}$, which also allows to develop redundant robot control experiments. Additionally the actuation system (direct drive) enables to configure $R^{3} \mathrm{D}$ as a haptic device. The first control experiments are done with Matlab Simulink environment using 
Real-Time Windows Target package which addresses control input and outputs via PCI DAQ on a PC.

As a result of the case control study, it is observed that the end-effector position accuracy is bounded in $\pm 1 \mathrm{~mm}$ for each axis through given trajectory. It should be noted that the controller is open for development and the accuracy may be enhanced in future studies with different control approaches. The design is devised to have the flexibility of using several control strategies either in joint-space or in task-space. In conclusion, demonstrations of the performances of classical controllers (such as PID controllers) on the robotic system can be given in undergraduate feedback control systems courses in both Mechanical and Electrical and Electronics Engineering programs at Izmir Institute of Technology. The robot can also be utilized to demonstrate several robotics and mechatronics concepts as part of the introductory robotics courses in the undergraduate curriculums of both Mechanical and Electrical and Electronics Engineering programs. The robot can serve as a test-bed for graduate level courses in the broad areas of control, robotics and mechatronics (such as the graduate level courses taught in Izmir Institute of Technology including: non-linear control, robot manipulator control, control of kinematically redundant robot manipulators, haptics and teleoperation). We plan to perform a survey similar to that of [28] as a future work for a larger set of students since this robot has to be tested by undergraduate and graduate students that are taking courses in the fields of control, teleoperation, and mechatronics.

\section{ACKNOWLEDGMENT}

This work is funded by The Scientific and Technological Research Council of Turkey via grant number 113E147.

\section{REFERENCES}

[1] A. Bayrak, F. Dogan, E. Tatlicioglu, and B. Ozdemirel, Design of an experimental twin-Rotor multi-Input multi-Output system, Comput App Eng Educ Arc 23 (2015), 578-586.

[2] S. Tuncer, Y. Tatar, and H. Guldemir, Design and implementation of an integrated environment for real-time control of power electronic systems, Comput Appl Eng Educ 17 (2009), 119-130.

[3] M. A. Khairurrijal and M. Budiman, Home-made PIC 16F877 microcontroller-based temperature control system for learning automatic control, Comput Appl Eng Educ 19 (2011), 10-17.

[4] V. F. Pires, J. F. Martins, and T. G. Amaral, Development of an experimental system for teaching induction motors with fault detection and diagnosis capabilities, Comput Appl Eng Educ 20 (2012), 611-618.

[5] E. Irmak, R. Bayindir, I. Colak, and M. Soysal, A remote laboratory experiment for 4-quadrant control of a DC motor, Comput Appl Eng Educ 19 (2011), 747-758.

[6] S. Reynard, O. Gomis-Bellmunt, A. Sudria-Andreu, O. BoixAragones, and I. Benitez-Pina, Flexible manufacturing cell SCADA system for educational purposes, Comput Appl Eng Educ 16 (2011), 21-30.

[7] M. T. Hagan and C. D. Latino, A modular control systems laboratory, Comput Appl Eng Educ 3 (1995), 89-96.
[8] C. Lafountain, K. Cohen, and S. Abdallah, Use of XFOIL in design of camber-controlled morphing UAVs, Comput Appl Eng Educ 20 (2012), 673-680.

[9] R. Isermann, Modeling and design methodology for mechatronic systems, IEEE/ASME Trans Mechatronics 1 (1996), 16-28.

[10] S. Omari, M.-D. Hua, G. Ducard, and T. Hamel, Hardware and software architecture for nonlinear control of multirotor helicopters, IEEE/ASME Trans Mechatronics 18 (2013), 1724-1736.

[11] M. L. Corradini, A. Cristofaro, F. Giannoni, and G. Orlando, Control systems with saturating inputs: Analysis tools and advanced design. Springer, London, UK, 2012.

[12] F. Mondada, M. Bonani, X. Raemy, J. Pugh, C. Cianci, A. Klaptocz, S. Magnenat, J.-C. Zufferey, D. Floreano, and A. Martinoli, "The epuck, a Robot Designed for Education in Engineering", Proceedings of the 9th Conference on Autonomous Robot Systems and Competitions. 1(1), 59-65, 2009.

[13] L.R. Soares and V.H.C. Alcalde, "An Educational Robotic Workstation based on the Rhino XR4 robot", Frontiers in Education Conference, 36th Annual, 7-12, 2006.

[14] M. Ueberle and M. Buss, Design, Control, and Evauation of a New 6 DOF Haptic Device, Proceedings of IEEE/RSJ International Conference on Intelligent Robots and System (IROSZOOZ), 1(3), 2002, pp 2949-2954.

[15] L. Birglen, C. Gosselin, N. Pouliot, B. Monsarrat, and T. Lalibert, SHaDe, a new 3-DOF haptic device, IEEE Transactions on Robotics and Automation 18 (2002), 166-175.

[16] R. Q. Van der Linde and P. Lammertse, HapticMaster-A generic force controlled robot for human interaction, Ind Robot 30 (2003), $515-52$.

[17] J. Arata, H. Kondo, M. Sakaguchi, and H. Fujoimoto, Development of a haptic device "DELTA-4" using parallel link mechanism. In: Proceedings International Conference Robot. Autom., 2009, pp 294-300.

[18] J. Arata, H. Kondo, M. Sakaguchi, and H. Fujoimoto, Haptics device using a newly development redundant parallel mechanism, IEEE Trans on Robotics 27 (2011), 201-214.

[19] F. L. Lewis, D. M. Dawson, and C. T. Abdallah, Robot manipulator vontrol: theory and Practice. Marcel Dekker, New York, NY, USA, 2003.

[20] M. W. Spong and M. Vidyasagar, Robot Dynamics and Control. John Wiley \& Sons Inc, New York, NY, USA, 1989.

[21] B. Siciliano and O. Khatib, Springer handbook of robotics. Springer, Secaucus, NJ, USA, 2008.

[22] E. S. Conkur and R. Buckingham, Clarifying the definition of redundancy as used in robotics. Robotica. Cambridge University Press, UK, 15 (1997), 583-586.

[23] S. Nicosia and P. Tomei, Robot control by using only joint position measurements, IEEE Tr on Automatic Control 35 (1990), 1058-1061.

[24] E. Tatlicioglu, M. L. McIntyre, D. M. Dawson, and I. D. Walker, Adaptive nonlinear tracking control of kinematically redundant robot manipulators, Int J Robot Autom 23 (2008), 98-105.

[25] E. Zergeroglu, D. Dawson, I. D. Walker, and A. Behal, Nonlinear tracking control of kinematically redundant robot manipulators. In: Proc, American Control Conf, Chicago, Il, USA, (2000), pp 2513-2517.

[26] E. Zergeroglu, D. M. Dawson, I. D. Walker, and P. Setlur, Nonlinear tracking control of kinematically redundant robot manipulators, IEEE/ASME Trans Mechatron 9 (2004), 129-132.

[27] P. Hsu, J. Hauser, and S. Sastry, Dynamic control of redundant manipulators, J Robot Syst 6 (1989), 133-148.

[28] A. Gil, O. Reinoso, J. M. Marin, L. Paya, and J. Ruiz, Development and deployment of a new robotics toolbox for education, Comput Appl Eng Educ 23 (2015), 443-454. 


\section{APPENDIX: ENTRIES OF THE DYNAMIC MODELING MATRICES}

The generalized inertia coefficients are included using terms represented in Equation 12 listed below:

$$
\begin{gathered}
m_{11}=I_{1}+I_{2}+I_{3}+m_{1} l_{1}^{2}+m_{2}\left(a_{1}^{2}+l_{2}^{2}+2 a_{1} l_{2} \cos \theta_{2}\right)+m_{3}\left(a_{1}^{2}+a_{2}^{2}+l_{3}^{2}+2 a_{1} a_{2} \cos \theta_{2}+2 a_{1} l_{3} \cos \theta_{23}+2 a_{2} l_{3} \cos \theta_{3}\right) \\
m_{22}=m_{2} l_{2}^{2}+m_{3}\left(a_{2}^{2}+l_{3}^{2}+2 a_{2} l_{3} \cos \theta_{3}\right)+I_{2}+I_{3} \\
m_{33}=m_{3} l_{3}^{2}+I_{3} \\
m_{12}=\left[m_{2}\left(a_{1} l_{2} \cos \theta_{2}+l_{2}^{2}\right)+\right. \\
\left.m_{3}\left(a_{1} a_{2} \cos \theta_{2}+a_{1} l_{3} \cos \theta_{23}+a_{2}^{2}+2 a_{2} l_{3} \cos \theta_{3}+l_{3}^{2}\right)+I_{2}+I_{3}\right] \\
m_{13}=\left[m_{3}\left(a_{1} l_{3} \cos \theta_{23}+a_{2} l_{3} \cos \theta_{3}+l_{3}^{2}\right)+I_{3}\right] \\
\\
m_{23}=m_{3}\left(a_{2} l_{3} \cos \theta_{3}+l_{3}^{2}\right)+I_{3}
\end{gathered}
$$

The centripetal and Coriolis coefficients are included using terms represented in Equation 12 listed below:

$$
\begin{gathered}
B_{12}=-m_{2} a_{1} l_{2} \sin \theta_{2}-m_{3}\left(a_{1} a_{2} \sin \theta_{2}+a_{1} l_{3} \sin \theta_{23}\right) \\
B_{13}=-m_{3}\left(a_{1} l_{3} \sin \theta_{23}+a_{2} l_{3} \sin \theta_{3}\right) \\
B_{21}=m_{2}\left(a_{1} l_{2} \sin \theta_{2}\right)+m_{3}\left(a_{1} a_{2} \sin \theta_{2}\right)+m_{3}\left(a_{1} l_{3} \sin \theta_{23}\right) \\
B_{23}=-m_{3}\left(a_{2} l_{3} \sin \theta_{3}\right) \\
B_{31}=m_{3} a_{1} l_{3} \sin \theta_{23}+m_{3} a_{2} l_{3} \sin \theta_{3} \\
B_{32}=m_{3} a_{2} l_{3} \sin \theta_{3} \\
\gamma_{112}=-2 m_{2} a_{1} l_{2} \sin \theta_{2}-2 m_{3} a_{1} a_{2} \sin \theta_{2}-2 m_{3} a_{1} l_{3} \sin \theta_{23} \\
\gamma_{113}=-2 m_{3} a_{1} l_{3} \sin \theta_{23}-2 m_{3} a_{2} l_{3} \sin \theta_{3} \\
\gamma_{123}=-m_{3} a_{1} l_{3} \sin \theta_{23}-2 m_{3} a_{2} l_{3} \sin \theta_{3}-m_{3} a_{1} l_{3} \sin \theta_{23} \\
\gamma_{213}=-2 m_{3} a_{2} l_{3} \sin \theta_{3} \\
\gamma_{223}=-2 m_{3} a_{2} l_{3} \sin \theta_{3} \\
\gamma_{312}=2 m_{3} a_{2} l_{3} \sin \theta_{3}
\end{gathered}
$$

\section{BIOGRAPHIES}

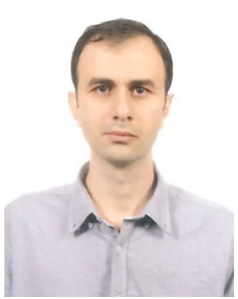

Osman Nuri Sahin received his BSc degree in Mechanical Engineering from Balikesir University, Balikesir, Turkey in 2005. Then, he received his MSc degree in Mechanical Engineering from Izmir Institute of Technology, Izmir, Turkey in 2012. Since 2013, he is pursuing his PhD degree at the Department of Mechanical Engineering at Izmir Institute of Technology. He is co-founder of NLFD Technology Inc. which focuses on robotic system design and applications at Izmir Technology Development Zone Inc., Izmir, Turkey. His research interests include mobile robotics, fault diagnosis and recovery of mobile robots, obstacle avoidance, haptic systems, and unlimited-workspace teleoperation.

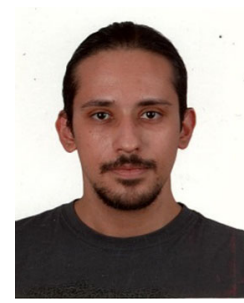

Emre Uzunoglu received his $\mathrm{BSc}$ and $\mathrm{MSc}$ degrees in Mechanical Engineering from Izmir Institute of Technology, Izmir, Turkey. Currently, he is pursuing the $\mathrm{PhD}$ degree at Izmir Institute of Technology. He is also co-founder of NLFD Technology Inc. which was established at Izmir Technology Development Zone Inc., Izmir, Turkey. His research interests include design and control of robotic systems, bilateral teleoperation, macro-micro manipulation, and 


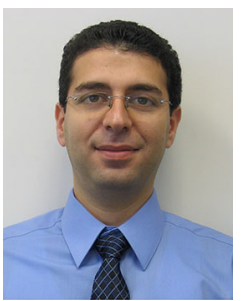

Enver Tatlicioglu received the BSc degree in Electrical \& Electronics Engineering from Dokuz Eylul University, Izmir, Turkey, and the $\mathrm{PhD}$ degree in Electrical and Computer Engineering from Clemson University, Clemson, SC, USA in 1999 and 2007, respectively. Upon completion of his PhD degree, he worked as a post-doctoral research fellow in the Department of Electrical and Computer Engineering at Clemson University then he joined the Department of Electrical \& Electronics Engineering at Izmir Institute of Technology, Izmir, Turkey where he is currently an associate professor. His research interests include control and identification of time delay systems, dynamic modelling of extensible continuum robot manipulators, non-linear control techniques for kinematically redundant robot manipulators, partial state feedback and output feedback control, haptic systems and teleoperation; learning, robust, and adaptive control of non-linear systems.

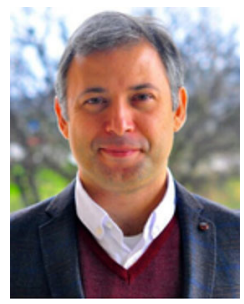

M. I. Can Dede received the BSc and MSc degrees in Mechanical Engineering from Istanbul Technical University (ITU, Istanbul, Turkey) and Middle East Technical University (METU, Ankara, Turkey) in 1999 and 2003, respectively. In 2007, he received the $\mathrm{PhD}$ degree from Department of Mechanical and Materials Engineering of Florida International University, Miami, FL, USA. From 2000 to 2003, he worked for ASELSAN Military Electronics Industries, Ankara, Turkey Microwave and Systems Technologies Division as Mechatronics Design Engineer. In 2009, after he received EU FP7 Marie-Curie fellowship to be re-integrated back to Europe, he joined the Mechanical Engineering Department of Izmir Institute of Technology, Izmir, Turkey as assistant professor. He was appointed as Vice Chair of Mechanical Engineering Department in between years 2010 and 2016. He has been a member of Executive Council of International Federation for Promotion of Mechanism and Machine Science (IFToMM) since 2016. His main research interests are in the fields of teleoperation systems, haptic device design, medical robotics, synthesis of spatial mechanisms, fault-tolerant system design, dynamic modeling, and controller design. 